\title{
Local dual spaces of a Banach space
}

\author{
by \\ Manuel GonzÁlez (Santander) \\ and Antonio Martínez-Abejón (Oviedo)
}

\begin{abstract}
We study the local dual spaces of a Banach space $X$, which can be described as the subspaces of $X^{*}$ that have the properties that the principle of local reflexivity attributes to $X$ as a subspace of $X^{* *}$.

We give several characterizations of local dual spaces, which allow us to show many examples. Moreover, every separable space $X$ has a separable local dual $Z$, and we can choose $Z$ with the metric approximation property if $X$ has it. We also show that a separable space containing no copies of $\ell_{1}$ admits a smallest local dual.
\end{abstract}

1. Introduction. The principle of local reflexivity [14] shows that there is a close relation between a Banach space $X$ and its second dual $X^{* *}$ from a finite-dimensional point of view: $X^{* *}$ is finitely dual representable in $X$ with $\varepsilon$-isometries that fix points (see Definition 2.1). This means that $X$ can be considered as a "local" dual of $X^{*}$.

In [9] the authors introduced the polar property as a test to check if $X^{*}$ is finitely dual representable in its subspaces. Here we consider a smaller class of subspaces $Z$ of $X^{*}$ (Definition 2.1) that satisfy the principle of local reflexivity in full force: $X^{*}$ is finitely dual representable in $Z$ with $\varepsilon$-isometries that fix points. So we can properly refer to these subspaces $Z$ as local dual spaces of $X$. We give several characterizations of such spaces, and we describe examples of local dual spaces for some classical spaces like $C[0,1], L_{1}[0,1]$, and for some families of Banach spaces, like $\ell_{1}\left(X^{*}\right), \ell_{\infty}(X)$, $X \otimes_{\pi} Y$ and $X \otimes_{\varepsilon} Y$ in the case that $Y^{*}$ has the metric approximation property (M.A.P., for short). We show that for $\mu$ a finite positive measure, $L_{1}\left(\mu, X^{*}\right)$ is a local dual of $L_{\infty}(\mu, X)$, improving a result of Díaz [3]. We also prove that every separable space with the M.A.P. has a separable local dual space with the M.A.P.

2000 Mathematics Subject Classification: Primary 46B10, 46B20; Secondary 46B04, 46B08.

Key words and phrases: local dual space, local reflexivity, norming subspace.

Research supported in part by DGICYT Grant PB 97-0349 (Spain). 
The relation between a Banach space $X$ and its local dual spaces is symmetric, in the sense that any local dual $Z$ of $X$ has a local dual isometric to $X$. This fact can also be seen as an extension of the local reflexivity principle.

We prove that every subspace $L$ of $X^{*}$ is contained in a local dual $Z$ of $X$ with $\operatorname{dens}(Z)=\max \{\operatorname{dens}(L), \operatorname{dens}(X)\}$. Using this fact and some results of Godefroy and Kalton [7], we show that a separable space $X$ containing no copies of $\ell_{1}$ admits a smallest local dual $Z_{d}$ which is also separable. This result provides an answer to a question in [7]. We also give a partial answer to another question in [7] by showing that a space $X$ isometric to a dual space has a smallest local dual $Z_{d}$ if and only if it admits a smallest norming subspace $Z_{n}$, and in this case $Z_{d}=Z_{n}$.

In the paper $X$ and $Y$ are Banach spaces, $B_{X}$ the closed unit ball of $X, S_{X}$ the unit sphere of $X$, and $X^{*}$ the dual of $X$. We identify $X$ with a subspace of $X^{* *}$. A subspace is always a closed subspace. For $A \subset X$ we consider the sets

$$
\begin{aligned}
A^{\circ} & :=\left\{f \in X^{*}:|\langle f, x\rangle| \leq 1 \text { for every } x \in A\right\}, \\
A^{\perp} & :=\left\{f \in X^{*}:\langle f, x\rangle=0 \text { for every } x \in A\right\} .
\end{aligned}
$$

Analogously, for $C \subset X^{*}$, we define the subsets $C_{\circ}$ and $C_{\perp}$ of $X$. We denote by $\mathcal{B}(X, Y)$ the space of all (bounded linear) operators from $X$ into $Y$, and by $\mathcal{K}(X, Y)$ the subspace of all compact operators. Given $T \in \mathcal{B}(X, Y)$, $N(T)$ and $R(T)$ are the range and the kernel of $T$, and $T^{*}$ is the conjugate operator of $T$.

Given a number $0<\varepsilon<1$, an operator $T \in \mathcal{B}(X, Y)$ is an $\varepsilon$-isometry if it satisfies $(1+\varepsilon)^{-1}<\|T x\|<1+\varepsilon$ for all $x \in S_{X}$. A space $X$ is said to be finitely representable in $Y$ if for each $\varepsilon>0$ and each finite-dimensional subspace $M$ of $X$ there is an $\varepsilon$-isometry $T: M \rightarrow Y$. We denote by $\mathbb{N}$ the set of all positive integers.

2. Local dual spaces. Recall that a subspace $Z$ of $X^{*}$ is norming if

$$
\|x\|=\sup \left\{|\langle f, x\rangle|: f \in B_{Z}\right\} \quad \text { for every } x \in X .
$$

Moreover, $X^{*}$ is finitely dual representable (f.d.r., for short) in $Z$ [9, Definition 1] if for every couple of finite-dimensional subspaces $F$ of $X^{*}$ and $G$ of $X$, and for every $0<\varepsilon<1$, there is an $\varepsilon$-isometry $L: F \rightarrow Z$ such that $\langle L f, x\rangle=\langle f, x\rangle$ for all $x \in G$ and all $f \in F$.

Clearly, if $X^{*}$ is f.d.r. in $Z$, then $Z$ is norming. However, the converse implication does not hold [9, Remark after Theorem 4].

Now we introduce a concept which is strictly stronger than finite dual representability (see Example 2.11). 
Definition 2.1. Let $Z$ be a subspace of $X^{*}$. We say that $Z$ is a local dual space of $X$ if for every couple of finite-dimensional subspaces $F$ of $X^{*}$ and $G$ of $X$, and every number $0<\varepsilon<1$, there is an $\varepsilon$-isometry $L: F \rightarrow Z$ satisfying the following conditions:

(a) $\langle L f, x\rangle=\langle f, x\rangle$ for all $x \in G$ and all $f \in F$, and

(b) $L f=f$ for all $f \in F \cap Z$.

Remark 2.2. (a) Obviously, $X^{*}$ is a local dual of $X$.

(b) A local dual of $X$ provides an almost-isometric local representation of $X^{*}$. This could be useful when we do not have a description of $X^{*}$, but it is possible to find a local dual. This happens for the ultrapowers $X_{\mathfrak{U}}$ of $X$ and for $L_{\infty}(\mu, X)$ (see (c) and Corollary 2.7).

(c) The principle of local reflexivity [14] establishes that a Banach space $X$ (as well as every isometric predual of $X^{*}$ ) is a local dual of $X^{*}$, and the principle of local reflexivity for ultrapowers [12, Theorem 7.3] establishes that $\left(X^{*}\right)_{\mathfrak{U}}$ is a local dual of $X_{\mathfrak{U}}$.

(d) There are spaces $X$ so that $X^{*}$ contains no proper norming subspaces. Hence $X^{*}$ is the only local dual of $X$. This is the case when $X$ is an Mideal in its bidual [11, Corollary III.2.16], or more generally, when $X$ is Hahn-Banach smooth. This means that every $x^{*} \in X^{*}$ admits only one Hahn-Banach extension to $X^{* *}[21]$.

For the structure of Banach spaces admitting no proper norming subspaces, we refer to [6], specially Theorem 8.3 where a characterization of these spaces is given, and [2].

The following technical result is a direct application of the Hahn-Banach Theorem and the principle of local reflexivity.

Lemma 2.3. Let $Z$ be a norming subspace of $X^{*}$. Then for every couple of finite-dimensional subspaces $E$ of $Z^{\perp \perp}$ and $F$ of $X$, and every $0<\varepsilon<1$, there is an $\varepsilon$-isometry $L: E \rightarrow Z$ such that $L e=e$ for all $e \in E \cap Z$, and $\langle$ Le,$x\rangle=\langle e, x\rangle$ for all $e \in E$ and all $x \in F$.

Proof. The canonical isometry $T: Z^{\perp \perp} \rightarrow Z^{* *}$ maps $f \in Z^{\perp \perp}$ to the functional $\widehat{f} \in Z^{* *}$ defined by $\langle\widehat{f}, \zeta\rangle:=\left\langle f, \zeta_{e}\right\rangle$, where $\zeta_{e} \in\left(X^{*}\right)^{*}$ is any HahnBanach extension of $\zeta \in Z^{*}$. Note that $\left\langle T^{-1} \widehat{f}, g\right\rangle:=\left\langle\widehat{f},\left.g\right|_{Z}\right\rangle$ for every $g \in X^{* *}$.

Let $E_{1}:=T(E)$. Since $Z$ is norming, the map that sends $x \in F$ to the functional $\widehat{x} \in Z^{*}$ given by $\langle\widehat{x}, z\rangle:=\langle z, x\rangle$ is an isometry. So we can apply the principle of local reflexivity to get an $\varepsilon$-isometry $\Lambda: E_{1} \rightarrow Z$ such that $\langle a, \widehat{x}\rangle=\langle\Lambda a, x\rangle$ for all $a \in E_{1}$ and $x \in F$, and $\Lambda a=a$ for all $a \in E_{1} \cap Z$. The $\varepsilon$-isometry $L:=\left.\Lambda T\right|_{E}$ satisfies the requirements of our statement.

Definition 2.4. Given a couple of subspaces $Z$ of $X^{*}$ and $G$ of $Z^{*}$, an operator $L: G \rightarrow X^{* *}$ is said to be an extension operator if $\left.L f\right|_{Z}=f$ for every $f \in G$. 
The following result will be very useful to find examples of local dual spaces of a Banach space $X$. The proof uses an ultrapower version of the Lindenstrauss compactness principle and some ideas of [15]. These ideas have also been used in [8, Proposition 3.6] in order to give a local characterization of subspaces of a Banach space which are unconditional ideals.

TheOREM 2.5. For a subspace $Z$ of $X^{*}$, the following statements are equivalent:

(1) $Z$ is a local dual of $X$;

(2) for every couple of finite-dimensional subspaces $F$ of $X^{*}$ and $G$ of $X$, and every $0<\varepsilon<1$, there is an $\varepsilon$-isometry $L: F \rightarrow Z$ such that

$\left(\mathrm{a}^{\prime}\right)|\langle L f, x\rangle-\langle f, x\rangle|<\varepsilon\|f\| \cdot\|x\|$ for all $x \in G$ and all $f \in F$, and $\left(\mathrm{b}^{\prime}\right)\|L f-f\| \leq \varepsilon\|f\|$ for all $f \in F \cap Z$;

(3) there is an isometric extension operator $L: Z^{*} \rightarrow X^{* *}$ so that $R(L) \supset X$;

(4) there exists a norm-one projection $P: X^{* *} \rightarrow X^{* *}$ such that $N(P)=$ $Z^{\perp}$ and $R(P) \supset X$;

(5) there exists a norm-one projection $Q: X^{* * *} \rightarrow X^{* * *}$ such that $R(Q)=Z^{\perp \perp}$ and $N(Q) \subset X^{\perp}\left(\right.$ where $\left.X^{* * *}=X^{*} \oplus X^{\perp}\right)$.

Proof. We denote by $\iota$ the natural inclusion operator from $Z$ into $X^{*}$. Observe that $\iota^{*}: X^{* *} \rightarrow Z^{*}$ is the restriction operator: $\iota^{*}(F)=\left.F\right|_{Z}$.

$(1) \Rightarrow(2)$. This is trivial.

$(2) \Rightarrow(3)$. First, for every compact operator $T: Z \rightarrow Y$ we obtain a compact extension $\widetilde{T}: X^{*} \rightarrow Y$ with $\|\widetilde{T}\|=\|T\|$, as follows:

Let $\mathcal{A}$ be the family of all pairs $\alpha=\left(E_{\alpha}, F_{\alpha}\right)$ of finite-dimensional subspaces $E_{\alpha} \subset X^{*}$ and $F_{\alpha} \subset X$. We define $|\alpha|:=\operatorname{dim} E_{\alpha}+\operatorname{dim} F_{\alpha}$. For every $\alpha \in \mathcal{A}$ we select an $|\alpha|^{-1}$-isometry $L_{\alpha}: E_{\alpha} \rightarrow Z$ such that $\left|\left\langle L_{\alpha} e, x\right\rangle-\langle e, x\rangle\right|<\varepsilon\|e\| \cdot\|x\|$ for all $e \in E_{\alpha}$ and all $x \in F_{\alpha}$, and $\left\|L_{\alpha} z-z\right\| \leq$ $|\alpha|^{-1}\|z\|$ for all $z \in E_{\alpha} \cap Z$.

We fix an ultrafilter $\mathfrak{U}$ on $\mathcal{A}$ refining the order filter associated to the order inclusion. Taking $L_{\alpha} g=0$ for $g \notin E_{\alpha}$, we can define the operator

$$
\widetilde{T} g:=\lim _{\alpha \rightarrow \mathfrak{U}} T L_{\alpha} g, \quad g \in X^{*} .
$$

Note that $\left(T L_{\alpha} g\right)_{\alpha \in \mathcal{A}}$ is contained in the compact set $2\|g\| \cdot \overline{T B_{Z}}$. Since $\left.L_{\alpha}\right|_{E_{\alpha} \cap Z}$ converges to the identity map, $\widetilde{T}$ is an extension of $T$; i.e., $\widetilde{T} \iota=T$. In particular, $\|T\| \leq\|\widetilde{T}\|$. Moreover, $\|\widetilde{T}\|=\lim _{\alpha \rightarrow \mathfrak{U}}\left\|T L_{\alpha}\right\| \leq\|T\|$.

Now, for every finite-dimensional subspace $G$ of $Z^{*}$, we consider the quotient map $q_{G}: Z \rightarrow Z / G_{\perp}$, and denote by $\iota_{G}$ the inclusion operator from $G$ into $Z^{*}$. Let $Q_{G}: X^{*} \rightarrow Z / G_{\perp}$ be the extension of $q_{G}$ built as in the first part of the proof. Note that $\iota^{*} Q_{G}^{*}=\iota_{G}$, so $Q_{G}^{*}: G \rightarrow X^{* *}$ is an isometric extension operator. 
Let $\mathfrak{V}$ be an ultrafilter on the set of all the finite-dimensional subspaces of $Z$, refining the filter associated to the order inclusion. The $w^{*}$-compactness of $B_{X^{* *}}$ allows us to define $L: Z^{*} \rightarrow X^{* *}$ as $L h:=w^{*}-\lim _{G \rightarrow \mathfrak{V}} Q_{G}^{*} h$. Clearly, $\|L\|=1$ and $\iota^{*} L$ is the identity, so $L$ is an extension operator.

It only remains to see that $R(L) \supset X$. Since every $x \in X$ belongs to $X^{* *}$, we can consider its restriction $\left.x\right|_{Z} \in Z^{*}$. Let $G$ be any finite-dimensional subspace of $Z^{*}$ containing $\left.x\right|_{Z}$. Then for every $f \in X^{*}$, we have

$$
\begin{aligned}
\left\langle Q_{G}^{*}\left(\left.x\right|_{Z}\right), f\right\rangle & =\left\langle\left. x\right|_{Z}, Q_{G} f\right\rangle=\lim _{\alpha \rightarrow \mathfrak{U}}\left\langle\left. x\right|_{Z}, q_{G} L_{\alpha} f\right\rangle \\
& =\lim _{\alpha \rightarrow \mathfrak{U}}\left\langle\iota_{G}\left(\left.x\right|_{Z}\right), L_{\alpha} f\right\rangle=\lim _{\alpha \rightarrow \mathfrak{U}}\left\langle L_{\alpha} f, x\right\rangle=\langle f, x\rangle,
\end{aligned}
$$

hence $Q_{G}^{*}\left(\left.x\right|_{Z}\right)=x$, so $x=L\left(\left.x\right|_{Z}\right)$, concluding the proof.

$(3) \Rightarrow(4)$. The operator $P:=L \iota^{*}$ defines a projection on $X^{* *}$, because $\iota^{*} L$ is the identity on $Z^{*}$, and $\|P\|=1$. Also, $R(P) \supset X$ since $\iota^{*}$ is surjective and $R(L) \supset X$. Finally, $N(P)=N\left(\iota^{*}\right)=Z^{\perp}$.

$(4) \Rightarrow(5)$. It is enough to take $Q=P^{*}$.

$(5) \Rightarrow(1)$. Let $Q$ be a norm-one projection on $X^{* * *}$ such that $R(Q)=Z^{\perp \perp}$ and $N(Q) \subset X^{\perp}$. First, considering the natural embedding of $X^{*}$ in $X^{* * *}$, we show that the restriction $\left.Q\right|_{X^{*}}$ is an isometry. Indeed, given $f \in X^{*}$ and $0<\varepsilon<1$, we select $x \in X$ such that $\|x\|=1$ and $\langle f, x\rangle>\|f\|-\varepsilon$. Since $R(I-Q) \subset X^{\perp}$, we have $\langle f, x\rangle=\langle Q f, x\rangle$; hence $\|Q f\|=\|f\|$.

Fix $F \in Z^{\perp}$ and $x \in X$. We choose $f \in X^{*}$ so that $\|f\|=1$ and $\langle f, x\rangle=\|x\|$. Since $R(Q)=Z^{\perp \perp}$ and $N(Q) \subset X^{\perp}$, we have

$$
\|F-x\| \geq|\langle F-x, Q f\rangle|=|\langle x, Q f\rangle|=\|x\| .
$$

By [5, Lemma I.1], we conclude that $Z$ is norming.

Now, in order to show that $Z$ is a local dual of $X$, we take a number $0<\varepsilon<1$ and finite-dimensional subspaces $E \subset X^{*}$ and $F \subset X$, and set $E_{1}:=Q(E) \subset Z^{\perp \perp}$.

Applying Lemma 2.3 , we get an $\varepsilon$-isometry $L: E_{1} \rightarrow Z$ so that

$$
\begin{array}{clrl}
\langle L e, x\rangle & =\langle e, x\rangle & & \text { for all } e \in E_{1}, x \in F, \\
L e & =e & & \text { for all } e \in E_{1} \cap Z .
\end{array}
$$

Thus $L Q: E \rightarrow Z$ is an $\varepsilon$-isometry. Moreover, given $e \in E \cap Z$, we have $L e=e$ and $Q e=e$, so $L Q e=e$. In addition, given $e \in E, x \in F$, we see that

$$
\langle L Q e, x\rangle=\langle Q e, x\rangle=\langle e, x\rangle,
$$

and the proof is complete.

Remark 2.6. Since $X$ is weak*-dense in $X^{* *}$, the projection $P$ in Theorem 2.5(4) cannot be weak*-continuous.

A direct application of Theorem 2.5 gives the following improvement of a result of Díaz [3, Theorem 2.1]. 
Corollary 2.7. Let $\mu$ be a finite positive measure. Then $L_{1}\left(\mu, X^{*}\right)$ is a local dual of $L_{\infty}(\mu, X)$.

Proof. It is enough to observe that, in the case that $\mu$ is a probability measure, [3, Theorem 2.1] establishes that $L_{1}\left(\mu, X^{*}\right)$ satisfies condition (2) in Theorem 2.5.

Next we apply Theorem 2.5 to show examples of local dual spaces for some classical Banach spaces.

Let $\lambda$ be a positive Borel measure on a metrizable compact space $K$. We denote by $B(K)$ the Banach algebra of all scalar, Borel-measurable bounded functions on $K$, endowed with the supremum norm. We can identify $L_{\infty}(\lambda)$ with the quotient $B(K) / \mathcal{J}_{0}$, where $\mathcal{J}_{0}:=\left\{f \in B(K): \int|f| d \lambda=0\right\}$. We denote by

$$
\pi_{\lambda}: B(K) \rightarrow L_{\infty}(\lambda)
$$

the canonical quotient map. Using the continuum hypothesis, it was proved in [18, Theorem 3], in the case $\operatorname{supp}(\lambda)=K$, that $\pi_{\lambda}$ admits a strong Borel lifting; i.e., there exists an algebra homomorphism

$$
\varrho_{\lambda}: L_{\infty}(\lambda) \rightarrow B(K)
$$

so that for every $f \in L_{\infty}(\lambda)$ we have $\varrho_{\lambda}(f)(t)=f(t)$ for $\lambda$-almost all $t \in K$, and $\varrho_{\lambda}(f)=f$ for every $f \in C(K)$.

It follows from these properties that $\varrho_{\lambda}$ is a right inverse of $\pi_{\lambda}$ that satisfies

$$
\left\|\varrho_{\lambda}(f)\right\|=\|f\|_{\infty} \quad \text { for every } f \in L_{\infty}(\lambda) .
$$

For $m$ the Lebesgue measure on $[0,1]$, the existence of $\varrho_{m}$ can be derived from the results of von Neumann and Stone in [19]. In this case we write $L_{\infty}[0,1]$ rather than $L_{\infty}(m)$. Recall that $L_{1}[0,1]^{*}=L_{\infty}[0,1]$ and $C[0,1]^{*}=$ $M[0,1]$, the space of all regular Borel measures on $[0,1]$. For every positive $\lambda \in M[0,1]$, the space $L_{1}(\lambda)$ is embedded in $M[0,1]$ through the map $f \mapsto$ $\lambda_{f}$, where $\lambda_{f}(U)=\int_{U} f d \lambda$.

Proposition 2.8. Assume the continuum hypothesis $2^{\omega}=\omega_{1}$.

(a) The natural copy of $C[0,1]$ in $L_{\infty}[0,1]$ is a local dual of $L_{1}[0,1]$.

(b) The natural copy of $L_{1}[0,1]$ in $M[0,1]$ is a local dual of $C[0,1]$.

Proof. (a) We consider the map $L: M[0,1] \rightarrow L_{\infty}[0,1]^{*}$ given by

$$
\langle L \mu, f\rangle:=\int_{0}^{1} \varrho_{m}(f)(t) d \mu(t) .
$$

This map is well defined because $\varrho_{m}(f)$ is Borel measurable for every $f$ in $L_{\infty}[0,1]$. Since $\varrho_{m}(g)=g$ for every $g \in C[0,1], L$ is an isometric extension operator from $C[0,1]^{*}$ into $L_{1}[0,1]^{* *}$. Moreover, $\varrho_{m}(f)(t)=f(t)$ a.e. for 
every $f$ implies that $L(h)=h$ for every $h \in L_{1}[0,1]$; hence $L(M[0,1]) \supset$ $L_{1}[0,1]$.

(b) The map $L_{m}: L_{\infty}[0,1] \rightarrow M[0,1]^{*}$ defined by

$$
\left\langle L_{m} f, \mu\right\rangle:=\int_{0}^{1} \varrho_{m}(f)(t) d \mu(t)
$$

is an isometric extension operator from $L_{1}[0,1]^{*}$ into $C[0,1]^{* *}$, because $M[0,1] \supset L_{1}[0,1]$ and $\varrho_{m}(f)(t)=f(t)$ a.e. for every $f$. Moreover, $\varrho_{m}(f)=f$ for every $f \in C[0,1]$ implies $L_{m}\left(L_{\infty}[0,1]\right) \supset C[0,1]$.

Remark 2.9. (a) In the proof of Proposition 2.8, we have applied the continuum hypothesis in order to select a Borel function for every $f \in L_{\infty}$, so that we can define an isometric extension operator $L: C[0,1]^{*} \rightarrow L_{\infty}[0,1]^{*}$. However, in our opinion it should be possible to find a proof in which the continuum hypothesis is not necessary.

(b) The Radon-Nikodym theorem allows us to write

$$
C[0,1]^{*}=L_{1}[0,1] \oplus_{1} M_{\text {sing }}[0,1] .
$$

So if $Q$ is the projection with range $L_{1}[0,1]$ and kernel $M_{\text {sing }}[0,1]$, then $Q^{*}$ is a norm-one projection on $C[0,1]^{* *}$ with $N\left(Q^{*}\right)=L_{1}[0,1]^{\perp}$. However, $R\left(Q^{*}\right)=M_{\text {sing }}[0,1]^{\perp} \not \supset C[0,1]$. Thus, we cannot apply part (4) of Theorem 2.5 to derive that $L_{1}[0,1]$ is a local dual of $C[0,1]$.

(c) Suppose that $\lambda \in M[0,1]$ is a positive Borel measure with support equal to $[0,1]$ and satisfying $m \perp \lambda$. Then using an argument similar to that in Proposition 2.8, we can prove that $L_{1}(\lambda)$ is a local dual of $C[0,1]$. Hence, $C[0,1]$ admits two local dual spaces with intersection $\{0\}$.

As an example of a measure $\lambda$ so that $L_{1}(\lambda) \cap L_{1}[0,1]=\{0\}$, we can consider the discrete measure associated to a dense sequence in $[0,1]$.

Proposition 2.8 and the principle of local reflexivity suggest that the relation of "being a local dual" is symmetric. Next we prove it.

Let $Z$ be a local dual of $X$. Denoting by $\widehat{x}$ the vector $x \in X$ viewed as an element of $X^{* *}$, we consider the following natural map:

$$
\Upsilon:\left.x \in X \mapsto \widehat{x}\right|_{Z} \in Z^{*} .
$$

Note that $\Upsilon$ is an isometry, because $Z$ is norming.

Proposition 2.10. Let $Z$ be a local dual of $X$ and let $L: Z^{*} \rightarrow X^{* *}$ be an isometric extension such that $L\left(Z^{*}\right) \supset X$. Then

(a) $L \Upsilon$ is the natural embedding from $X$ into $X^{* *}$.

(b) $\Upsilon(X)$ is a local dual of $Z$ isometric to $X$.

Proof. (a) Let $J$ and $\iota$ denote the embedding of $X$ in $X^{* *}$ and the embedding of $Z$ in $X^{*}$, respectively. Then $\Upsilon=\iota^{*} J$. Moreover, for every 
$z \in Z$ and $z^{*} \in Z^{*}$

$$
\left\langle\iota^{*} L z^{*}, z\right\rangle=\left\langle L z^{*}, \iota z\right\rangle=\left\langle z^{*}, z\right\rangle ;
$$

hence $\iota^{*} L$ is the identity on $Z^{*}$. Thus, $L \iota^{*}$ is a projection on $X^{* *}$ with $R\left(L \iota^{*}\right)=R(L) \supset X$; hence $L \Upsilon=L \iota^{*} J=J$.

(b) We define $\Lambda: \Upsilon(X)^{*} \rightarrow Z^{* *}$ by

$$
\langle\Lambda f, g\rangle:=\langle L g, f \circ \Upsilon\rangle, \quad f \in \Upsilon(X)^{*}, g \in Z^{*} .
$$

Clearly $\|\Lambda\| \leq 1$. Moreover, for every $f \in \Upsilon(X)^{*}$ and every $\Upsilon x \in \Upsilon(X)$,

$$
\langle\Lambda f, \Upsilon x\rangle=\langle L \Upsilon x, f \circ \Upsilon\rangle=\langle f \circ \Upsilon, x\rangle=\langle f, \Upsilon x\rangle .
$$

Thus $\Lambda$ is an isometric extension operator. Moreover, for every $y \in Z$ we can write $y=f \circ \Upsilon$ with $f \in \Upsilon(X)^{*}$. Then

$$
\langle\Lambda f, g\rangle=\langle L g, f \circ \Upsilon\rangle=\langle L g, y\rangle=\langle g, y\rangle
$$

for every $g \in Z^{*}$; hence $\Lambda\left(\Upsilon(X)^{*}\right) \supset Z$.

Now we show that $X^{*}$ f.d.r. in $Z$ does not imply that $Z$ is a local dual of $X$. In order to do that, observe that

$$
Z_{1} \subset Z_{2} \subset X^{*} \text { and } X^{*} \text { f.d.r. in } Z_{1} \Rightarrow X^{*} \text { f.d.r. in } Z_{2} \text {. }
$$

The following example shows that this implication is not valid for local dual spaces.

EXAMPLE 2.11. The principle of local reflexivity implies that $\ell_{\infty}$ is f.d.r. in $c_{0}$.

On the other hand, since the quotient map $q: \ell_{\infty} \rightarrow \ell_{\infty} / c_{0}$ is not weakly compact, $\ell_{\infty} / c_{0}$ contains a complemented subspace isomorphic to $\ell_{\infty}[17$, Proposition 2.f.4]. Therefore, there is a subspace $N$ of $\ell_{\infty} / c_{0}$ such that the quotient space $\left(\ell_{\infty} / c_{0}\right) / N$ is isomorphic to $\ell_{2}$. We take $M:=q^{-1}(N)$.

Claim. $\ell_{1}^{*}=\ell_{\infty}$ is f.d.r. in $M$, but $M$ is not a local dual of $\ell_{1}$.

Note that $\ell_{\infty} / M$ is isomorphic to $\left(\ell_{\infty} / c_{0}\right) / N$, so $M^{\perp}$ is isomorphic to $\ell_{2}$. Since $c_{0} \subset M$, we see that $\ell_{\infty}$ is f.d.r. in $M$. But $M^{\perp}$ is non-complemented in $\ell_{\infty}^{*}$ because $\ell_{\infty}^{*}$ has the Dunford-Pettis property. Therefore, by Theorem 2.5, $M$ is not a local dual of $\ell_{1}$.

Proposition 2.12. Let $X$ be a Banach space. Then

(a) $\ell_{1}\left(X^{*}\right)$ is a local dual of $\ell_{\infty}(X)$, and

(b) $\ell_{\infty}(X)$ is a local dual of $\ell_{1}\left(X^{*}\right)$.

Proof. (a) For every couple $\alpha:=(E, F)$ of finite-dimensional subspaces of $\ell_{1}\left(X^{*}\right), \ell_{\infty}\left(X^{* *}\right)$, we select a pair of sequences $\left(E_{n}\right),\left(F_{n}\right)$ of finitedimensional subspaces of $X^{*}$ and $X^{* *}$ respectively so that $E \subset \ell_{1}\left(E_{n}\right)$ and $F \subset \ell_{\infty}\left(F_{n}\right)$. We define $|\alpha|:=\operatorname{dim}(E)+\operatorname{dim}(F)$. 
The principle of local reflexivity allows us to find, for every $n$, an $|\alpha|^{-1}$ isometry $S_{n}^{\alpha}: F_{n} \rightarrow X$ so that $\left\langle S_{n}^{\alpha} f, e\right\rangle=\langle e, f\rangle$ for every $e \in E_{n}$ and $f \in F_{n}$, and $S_{n}^{\alpha}(f)=f$ for every $f \in F_{n} \cap X$. We consider the (non-linear) map $S^{\alpha}: \ell_{\infty}\left(X^{* *}\right) \rightarrow \ell_{\infty}(X)$ given by $S^{\alpha}\left(z_{n}\right):=\left(S_{n}^{\alpha}\left(z_{n}\right)\right)$ if $\left(z_{n}\right) \in F$, and $S^{\alpha}\left(z_{n}\right):=0$ otherwise.

Let $\mathfrak{U}$ be an ultrafilter in the set of all couples $\alpha=(E, F)$ of finitedimensional subspaces $E$ of $\ell_{1}\left(X^{*}\right)$ and $F$ of $\ell_{\infty}\left(X^{* *}\right)$ refining the order filter. We define an operator $\Lambda: \ell_{1}\left(X^{*}\right)^{*}=\ell_{\infty}\left(X^{* *}\right) \rightarrow \ell_{\infty}(X)^{* *}$ by

$$
\Lambda\left(\left(z_{n}\right)\right):=w^{*}-\lim _{\alpha \rightarrow \mathfrak{U}} S^{\alpha}\left(z_{n}\right), \quad\left(z_{n}\right) \in \ell_{\infty}\left(X^{* *}\right) .
$$

Note that $\Lambda$ is an isometry and $\Lambda\left(y_{n}\right)=\left(y_{n}\right)$ for every $\left(y_{n}\right) \in \ell_{\infty}\left(X^{* *}\right)$. Therefore, $\Lambda$ is an isometric extension operator. Moreover, $\Lambda\left(\left(x_{n}\right)\right)=\left(x_{n}\right)$ if $\left(x_{n}\right) \in \ell_{\infty}(X)$. In particular $\Lambda\left(\ell_{\infty}\left(X^{* *}\right)\right) \supset \ell_{\infty}(X)$.

(b) It is enough to observe that the operator $\Upsilon: \ell_{\infty}(X) \rightarrow \ell_{1}\left(X^{*}\right)^{*}$, introduced in Proposition 2.10, is the natural inclusion.

Recall that a Banach space $X$ has the metric approximation property (M.A.P., for short) if for every $\varepsilon>0$ and every compact set $K$ in $X$, there is a finite rank operator $T$ on $X$ such that $\|T\| \leq 1$ and $\|T x-x\| \leq \varepsilon$ for every $x \in K$. Note that if $X^{*}$ has the M.A.P., then so does $X[4$, Corollary VIII.3.9]. However, the converse implication is not valid [17, Theorem 1.e.7].

The following result is proved using some ideas of [13].

Proposition 2.13. Assume that $X^{*}$ or $Y^{*}$ has the M.A.P. Then

(a) $X^{*} \otimes_{\varepsilon} Y^{*}$ is a local dual of $X \otimes_{\pi} Y$, and

(b) $X^{*} \otimes_{\pi} Y^{*}$ is a local dual of $X \otimes_{\varepsilon} Y$.

Proof. We assume that $Y^{*}$ has the M.A.P.

(a) The dual space $\left(X \otimes_{\pi} Y\right)^{*}$ can be identified with $\mathcal{B}\left(X, Y^{*}\right)$. Moreover, since $Y^{*}$ has the M.A.P., $X^{*} \otimes_{\varepsilon} Y^{*}$ can be identified with $\mathcal{K}\left(X, Y^{*}\right)$, and there exists a net $\left(A_{\alpha}\right)$ of finite rank operators on $Y^{*}$ with $\left\|A_{\alpha}\right\| \leq 1$ so that $\lim _{\alpha}\left\|A_{\alpha} g-g\right\|=0$ for every $g \in Y^{*}$. We can assume that $\left(A_{\alpha}\right)$ is $\sigma\left(\mathcal{K}\left(Y^{*}\right)^{* *}, \mathcal{K}\left(Y^{*}\right)^{*}\right)$-convergent.

For $T \in \mathcal{B}\left(X, Y^{*}\right)$ and $\Phi \in \mathcal{K}\left(X, Y^{*}\right)^{*}$, the expression $\Phi_{T}(A):=\Phi(A T)$ defines $\Phi_{T} \in \mathcal{K}\left(Y^{*}\right)^{*}$. Then we define $\Lambda: \mathcal{K}\left(X, Y^{*}\right)^{*} \rightarrow \mathcal{B}\left(X, Y^{*}\right)^{*}$ by

$$
\langle\Lambda \Phi, T\rangle:=\lim _{\alpha}\left\langle\Phi, A_{\alpha} T\right\rangle=\lim _{\alpha}\left\langle A_{\alpha}, \Phi_{T}\right\rangle .
$$

Note that for every $f \otimes g \in X^{*} \otimes_{\varepsilon} Y^{*}$ we have

$$
\langle\Lambda \Phi, f \otimes g\rangle=\lim _{\alpha}\left\langle\Phi, A_{\alpha}(g) \cdot f\right\rangle=\langle\Phi, f \otimes g\rangle .
$$

So $\Lambda$ is an isometric extension operator. Analogously, we can check that for every $x \otimes y \in X \otimes_{\pi} Y \subset \mathcal{B}\left(X, Y^{*}\right)^{*}$, we have $\Lambda\left(\left.x \otimes y\right|_{\mathcal{K}\left(X, Y^{*}\right)}\right)=x \otimes y$. Thus $X \otimes_{\pi} Y \subset \Lambda\left(\mathcal{K}\left(X, Y^{*}\right)^{*}\right)$, and it is enough to apply Theorem 2.5. 
(b) The proof is analogous, identifying $\left(X \otimes_{\varepsilon} Y\right)^{*}$ with the space $\mathcal{I}\left(X, Y^{*}\right)$ of all integral operators from $X$ into $Y^{*}$.

Remark 2.14. (a) If we assume in Proposition 2.13 that $Y^{*}$ has the metric compact approximation property (defined as the M.A.P., but using compact operators instead of finite rank operators), then we find that $\mathcal{K}\left(X, Y^{*}\right)$ is a local dual of $X \otimes_{\pi} Y$.

(b) It follows from the results of Lima [16, Theorem 13] that if $Y^{*}$ has the Radon-Nikodym property and $Y^{* *} \otimes_{\varepsilon} Y^{*}$ is a local dual of $Y^{*} \otimes_{\pi} Y$, then $Y^{*}$ has the M.A.P. So it is not enough to assume in Proposition 2.13 that $X$ or $Y$ has the M.A.P.

(c) Let $\mu$ be a finite positive measure and let $K$ be a compact space. Since the spaces $L_{1}(\mu)^{*} \equiv L_{\infty}(\mu)$ and $C(K)^{*} \equiv M(K)$ have the M.A.P., it follows from Proposition 2.13 that $X^{*} \otimes_{\varepsilon} L_{\infty}(\mu)$ is a local dual of $L_{1}(\mu, X)=$ $X \otimes_{\pi} L_{1}(\mu)$, and that $X^{*} \otimes_{\pi} M(K)$ is a local dual of $C(K, X)=X \otimes_{\varepsilon} C(K)$.

(d) The tensor product $X^{*} \otimes_{\varepsilon} L_{\infty}(\mu)$ in part (c) can be identified with a (proper, in general) subspace of $L_{\infty}\left(\mu, X^{*}\right)$.

It has been proved in [10] that $L_{\infty}\left(\mu, X^{*}\right)$ is also a local dual of $L_{1}(\mu, X)$.

Casazza and Kalton [1] proved that for every separable Banach space $X$ with the M.A.P., we can find a sequence $\left(T_{n}\right)$ of finite rank operators on $X$ such that

(a) $\lim _{n \rightarrow \infty}\left\|T_{n} x-x\right\|=0$ for all $x \in X$,

(b) $\lim _{n \rightarrow \infty}\left\|T_{n}\right\|=1$ and

(c) $T_{n} T_{k}=T_{k} T_{n}=T_{\min \{k, n\}}$;

i.e., $X$ admits a commuting 1-approximating sequence $\left(T_{n}\right)$. Using this fact we show in the following result that a separable Banach space with the M.A.P. admits a local dual of $X$ with the M.A.P. Its proof is similar to the proof of [7, Lemma II.2].

Theorem 2.15. Let $X$ be a separable Banach space with the M.A.P., and let $\left(T_{n}\right)$ be a commuting 1-approximating sequence on $X$. Then $\overline{\bigcup_{n=1}^{\infty} R\left(T_{n}^{*}\right)}$ is a local dual of $X$, and has the M.A.P.

Proof. Let $\mathfrak{U}$ be an ultrafilter on $\mathbb{N}$. We define a map $P$ on $X^{* *}$ by

$$
P z:=w^{*}-\lim _{k \rightarrow \mathfrak{U}} T_{k}^{* *} z, \quad z \in X^{* *} .
$$

From $T_{n}^{* *} T_{k}^{* *}=T_{k}^{* *} T_{n}^{* *}=T_{\min \{k, n\}}^{* *}$ and the weak*-continuity of the operators $T_{n}^{* *}$, it follows that for every $n \in \mathbb{N}$ and every $z \in X^{* *}$, we have

$$
T_{n}^{* *} P z=P T_{n}^{* *} z=T_{n}^{* *} z .
$$

Hence $P^{2} z=w^{*}-\lim _{n \rightarrow \mathfrak{U}} T_{n}^{* *} P z=P z$. Since $\lim _{n \rightarrow \infty}\left\|T_{n}\right\|=1, P$ is a norm-one projection. Also, it follows from formula (1) that $N\left(T_{n}^{* *}\right) \supset N(P)$ 
for every $n \in \mathbb{N}$. Since the intersection of the kernels $N\left(T_{n}^{* *}\right)$ is clearly contained in $N(P)$, we get

$$
N(P)=\bigcap_{n=1}^{\infty} N\left(T_{n}^{* *}\right) .
$$

As a consequence, $N(P)$ is weak ${ }^{*}$-closed. And clearly $P\left(X^{* *}\right) \supset X$.

Note that $T_{n} T_{k}=T_{k} T_{n}=T_{\min \{k, n\}}$ implies $N\left(T_{n}^{* *}\right)_{\perp}=R\left(T_{n}^{*}\right) \subset$ $R\left(T_{n+1}^{*}\right)$ for every $n$. Therefore

$$
N(P)_{\perp}=\overline{\bigcup_{n=1}^{\infty} R\left(T_{n}^{*}\right),}
$$

and it follows from Theorem 2.5 that $\overline{\bigcup_{n=1}^{\infty} R\left(T_{n}^{*}\right)}$ is a local dual of $X$.

Moreover, since $T_{n}^{*} f$ is weak*-convergent for every $f \in X^{*}$, and compact operators take weak*-convergent sequences to norm-convergent sequences, by formula (1) we have $\lim _{k \rightarrow \infty}\left\|T_{k}^{*} f-f\right\|=\lim _{k \rightarrow \infty}\left\|T_{n}^{*}\left(T_{k}^{*} g-g\right)\right\|=0$ for every $f=T_{n}^{*} g \in R\left(T_{n}^{*}\right)$. Since $\left(T_{k}^{*}\right)$ is bounded, we get $\lim _{k \rightarrow \infty}\left\|T_{k}^{*} f-f\right\|$ $=0$ for every $f \in \overline{\bigcup_{n=1}^{\infty} R\left(T_{n}^{*}\right)}$; hence $\overline{\bigcup_{n=1}^{\infty} R\left(T_{n}^{*}\right)}$ has the M.A.P.

REMARK 2.16. If $X$ has a monotone Schauder basis, then the local dual of $X$ provided by Theorem 2.15 is the subspace generated by the coefficient functionals of the basis.

As an application of Theorem 2.15, we give another example of a local dual space of $L_{1}[0,1]$.

EXAMPLE 2.17. The subspace $Z$ of $L_{\infty}[0,1]$ generated by the characteristic functions $\chi_{n, i}$ of the dyadic intervals

$$
\left[\frac{i-1}{2^{n}}, \frac{i}{2^{n}}\right], \quad n=0,1,2, \ldots ; i=1, \ldots 2^{n},
$$

is a local dual of $L_{1}[0,1]$ isometric to $C(\Delta)$, where $\Delta$ denotes the Cantor set.

It is enough to check that the sequence $\left(P_{n}\right)$ of projections defined by

$$
P_{n} f:=\sum_{i=1}^{2^{n}}\left\langle 2^{n} \chi_{n, i}, f\right\rangle \chi_{n, i}
$$

is a commuting 1-approximating sequence in $L_{1}[0,1]$, and that $\bigcup_{n} R\left(P_{n}^{*}\right)$ is the subspace generated by the functions $\chi_{n, i}$.

In relation to the necessity of the continuum hypothesis in Proposition 2.8 , note that $C(\Delta)$ is isomorphic, but not isometric to $C[0,1]$.

We have seen in part (a) of the previous example that there are local dual spaces $Z_{1}$ and $Z_{2}$ of $C[0,1]$ so that $Z_{1} \cap Z_{2}$ is finite-dimensional. Now we will show that this cannot happen for spaces that contain no copies of $\ell_{1}$. 
Godefroy and Kalton [7] considered the family $\mathcal{P}_{X}$ of all the subspaces $Y$ of $X^{* *}$ for which there is a norm-one projection on $X^{* *}$ such that $Y=N(P)$ and $R(P) \supset X$. The following result is an application of [7, Proposition V.1] and our previous results.

Proposition 2.18. If $X$ contains no copies of $\ell_{1}$, then it admits a smallest local dual; i.e., there exists a local dual $Z_{d}$ contained in every local dual of $X$.

Proof. If $X$ contains no copies of $\ell_{1}$, then $\mathcal{P}_{X}$ consists of weak ${ }^{*}$-closed subspaces of $X^{* *}$ and has a largest element $L$ [7, Proposition V.1]. By Theorem 2.5, the local dual spaces of $X$ are precisely the subspaces $Z$ of $X^{*}$ such that $Z^{\perp} \in \mathcal{P}_{X}$. Thus $Z_{d}:=L_{\perp}$ is the smallest local dual of $X$.

The following result was obtained by Sims and Yost [20] (see [11, Lemmas III.4.3 and III.4.4]). Here, dens $(X)$ stands for the density character of $X$, defined as the smallest cardinal $\kappa$ for which $X$ has a dense subset of cardinality $\kappa$.

Proposition 2.19. Let $L$ be a subspace of $Y$, and let $F$ be a subspace of $Y^{*}$ with $\operatorname{dens}(F) \leq \operatorname{dens}(L)$. Then there exists a subspace $M$ of $Y$ with $\operatorname{dens}(M)=\operatorname{dens}(L)$ and $M \supset L$ for which there exists an isometric extension operator $T: M^{*} \rightarrow Y^{*}$ such that $T\left(M^{*}\right) \supset F$.

We now prove our next result about the existence of local dual spaces.

Proposition 2.20. Every subspace $L$ of $X^{*}$ is contained in a local dual $Z_{L}$ of $X$ with $\operatorname{dens}\left(Z_{L}\right)=\max \{\operatorname{dens}(L), \operatorname{dens}(X)\}$.

Proof. Given a subspace $L$ of $X^{*}$, it is easy to find a subspace $L_{0}$ of $X^{*}$ so that $L \subset L_{0}$ and $\operatorname{dens}\left(L_{0}\right)=\max \{\operatorname{dens}(L)$, dens $(X)\}$. If we apply Proposition 2.19 to $L_{0}$ as a subspace of $X^{*}$ and $X$ as a subspace of $X^{* *}$ we get a subspace $Z_{L}$ of $X^{*}$ with $Z_{L} \supset L$ and $\operatorname{dens}\left(Z_{L}\right)=\max \{\operatorname{dens}(L), \operatorname{dens}(X)\}$ for which there exists an isometric extension operator $T: Z_{L}^{*} \rightarrow X^{* *}$ such that $T\left(Z_{L}^{*}\right) \supset X$. By Theorem 2.5, this is the desired local dual of $X$.

Remark 2.21. (a) Assume that $X$ is separable and contains no copies of $\ell_{1}$, and that $X^{*}$ is not separable. By Proposition 2.20, the smallest local dual space $Z_{d}$ provided by Proposition 2.18 is separable; in particular, $Z_{d} \neq X^{*}$. This fact gives an affirmative answer to a question of Godefroy and Kalton in [7, Remarks V.3].

(b) Assume that $X$ contains no copies of $\ell_{1}$. In this case, apart from the smallest local dual $Z_{d}$ there also exists a smallest norming subspace $Z_{n} \subset X^{*}$ [5, Lemma I.2 and Theorem II.3]. Clearly $Z_{n}$ is contained in $Z_{d}$. However, we do not know whether or not $Z_{n}=Z_{d}$.

Question [7, Remarks V.3]. Assume that both $Z_{n}$ and $Z_{d}$ exist for $X$. Is $Z_{n}=Z_{d}$ ? 
We can only give an affirmative answer for dual spaces.

Proposition 2.22. Assume that $X$ is isometric to a dual space. Then $X$ admits a smallest local dual $Z_{d}$ if and only if it admits a smallest norming subspace $Z_{n}$. In this case $Z_{d}=Z_{n}$, and this space is the unique isometric predual of $X$.

Proof. By [5, Lemma I.2], the smallest norming subspace $Z_{n}$ exists if and only if

$$
Z_{n}^{\perp}=\left\{z \in X^{* *}:\|z-x\| \geq\|x\| \text { for every } x \in X\right\} .
$$

In this case $X^{* *}=X \oplus Z_{n}^{\perp}$ and $Z_{n}$ is the unique predual of $X$ [5, Theorem II.1].

Clearly, the projection $P$ on $X^{* *}$ with kernel $Z_{n}^{\perp}$ and range $X$ satisfies $\|P\|=1$ and the remaining conditions in Theorem 2.5. Hence $Z_{n}$ is a local dual of $X$, and it is the smallest one, because every local dual is norming.

Conversely, assume that the smallest local dual $Z_{d}$ exists, and let $P$ be the associated projection. If $X_{*}$ is a predual of $X$, then $X^{* *}=X \oplus X_{*}^{\perp}=$ $P(X) \oplus Z_{d}^{\perp}$. Since $X \subset P(X)$ and $Z_{d} \subset X_{*}$ (hence $X_{*}^{\perp} \subset Z_{d}^{\perp}$ ), we conclude that $Z_{d}=X_{*}$ and $X_{*}^{\perp}=Z_{d}^{\perp}$. In particular,

$$
Z_{d}^{\perp}=\left\{z \in X^{* *}:\|z-x\| \geq\|x\| \text { for every } x \in X\right\} ;
$$

hence $Z_{d}$ is the smallest norming subspace of $X$.

Remark 2.23. (a) In Proposition 2.22, we have seen that a dual space admitting a smallest norming subspace has a unique predual. However, this condition is not sufficient, since $L_{\infty}[0,1]$ has a unique predual but it does not admit a smallest norming subspace [5, Proposition IV.2].

(b) There are spaces $X$ containing no copies of $\ell_{1}$ so that $X^{*}$ is f.d.r. in a subspace $Z$ which is not a local dual of $X$.

Indeed, let $Y$ be a separable space such that $Y^{* *} / Y$ is isomorphic to $c_{0}$, and let $Q: Y^{* *} \rightarrow Y^{* *} / Y$ denote the quotient map. We select a subspace $M$ of $c_{0}$ such that $M^{\perp}$ is not complemented in $\ell_{1}$. For example, we can take $M$ so that $M^{\perp}$ is isomorphic to $\ell_{1}\left(\ell_{2}^{n}\right)$.

The space $X=Y^{*}$ contains no copies of $\ell_{1}$, and $X^{*}$ is f.d.r. in $Z:=$ $Q^{-1}(M)$, because $Y$ is contained in $Z$. However, $Z$ is not a local dual of $X$ because $Z^{\perp}=M^{\perp}$ is not complemented.

Acknowledgements. We are grateful to the referee for several valuable comments.

\section{References}

[1] P. G. Casazza and N. J. Kalton, Notes on approximation properties in separable Banach spaces, in: London Math. Soc. Lecture Note Ser. 158, Cambridge Univ. Press, 1990, 49-63. 
[2] D. Chen and B.-L. Li, Ball topology of Banach spaces, Houston J. Math. 22 (1996), 821-833.

[3] S. Díaz, A local approach to functionals on $L^{\infty}(\mu, X)$, Proc. Amer. Math. Soc. 128 (2000), 101-109.

[4] J. Diestel and J. J. Uhl, Jr., Vector Measures, Math. Surveys 15, Amer. Math. Soc., Providence, 1977.

[5] G. Godefroy, Existence and uniqueness of isometric preduals: a survey, in: Contemp. Math. 85, Amer. Math. Soc., 1989, 131-193.

[6] G. Godefroy and N. J. Kalton, The ball topology and its applications, ibid., 195-237.

[7] —, -, Approximating sequences and bidual projections, Quart. J. Math. Oxford 48 (1997), 179-202.

[8] G. Godefroy, N. J. Kalton and P. D. Saphar, Unconditional ideals in Banach spaces, Studia Math. 104 (1993), 13-59.

[9] M. González and A. Martínez-Abejón, Local reflexivity of dual Banach spaces, Pacific J. Math. 56 (1999), 263-278.

[10] —, - Local dual spaces of Banach spaces of vector-valued functions, preprint.

[11] P. Harmand, D. Werner and W. Werner, M-Ideals in Banach Spaces and Banach Algebras, Lecture Notes in Math. 1547, Springer, Berlin, 1993.

[12] S. Heinrich, Ultraproducts in Banach space theory, J. Reine Angew. Math. 313 (1980), 72-104.

[13] J. Johnson, Remarks on Banach spaces of compact operators, J. Funct. Anal. 32 (1979), 304-311.

[14] W. B. Johnson, H. P. Rosenthal and M. Zippin, On bases, finite dimensional decompositions and weaker structures in Banach spaces, Israel J. Math. 9 (1971), $488-506$.

[15] N. J. Kalton, Locally complemented subspaces and $\mathcal{L}_{p}$-spaces for $0<p<1$, Math. Nachr. 115 (1984), 71-97.

[16] Å. Lima, The metric approximation property, norm-one projections and intersection properties of balls, Israel J. Math. 84 (1993), 451-475.

[17] J. Lindenstrauss and L. Tzafriri, Classical Banach Spaces I. Sequence Spaces, Springer, 1977.

[18] S. P. Lloyd, Two lifting theorems, Proc. Amer. Math. Soc. 42 (1974), 128-134.

[19] J. von Neumann and M. H. Stone, The determination of representative elements in the residual classes of a Boolean algebra, Fund. Math. 25 (1935), 353-378.

[20] B. Sims and D. Yost, Banach spaces with many projections, Proc. Centre Math. Anal. Austral. Nat. Univ. 14 (1986), 335-342.

[21] F. Sullivan, Geometrical properties determined by the higher duals of a Banach space, Illinois J. Math. 21 (1977), 315-331.

Departamento de Matemáticas

Facultad de Ciencias

Universidad de Cantabria

E-39071 Santander, Spain

E-mail: gonzalem@ccaix3.unican.es
Departamento de Matemáticas Facultad de Ciencias Universidad de Oviedo E-33007 Oviedo, Spain E-mail: ama@pinon.ccu.uniovi.es 